\title{
Multidisciplinary Design Optimization of UAV Airframes
}

\author{
András Sóbester*, Andy J. Keane ${ }^{\dagger}$ \\ University of Southampton, Southampton, Hampshire, SO17 1BJ, UK
}

\begin{abstract}
If one considers the problem of converting an aircraft mission profile into an airframe design from an optimization theory perspective, it becomes obvious that the search problem comes with all the trimmings. The design space is large and multidimensional, there are multiple and often highly multimodal objectives and constraints, these depending not only on the design variables, but often on each other as well. Multidisciplinary Design Optimization studies can be conducted at different levels of detail, depending on the chosen tradeoff between the size of the design space and the fidelity of the analysis. In this paper we discuss some of the challenges arising at the conceptual level, where simple, but versatile models and low cost analysis tools are used to guide the designer through the first, fundamental decisions of the design process. At the center of our proposed design workflow lies a parametric geometry, residing in an off-the-shelf Computer-Aided Design (CAD) tool - this provides the models required by the multidisciplinary analyses. We also touch on some of the issues specific to the design of our chosen class of aircraft - Unmanned Air Vehicles (UAVs). To summarize: a CAD-based UAV conceptual design framework is proposed and demonstrated.
\end{abstract}

\section{Introduction}

IRCRAFT design requires input from a variety of disciplines - it is, to quote Stinton, ${ }^{1}$ " $a$
mixture of precise science, disciplined methods, consummate accuracy in telling it as it is after a test flight, gut feeling and artistry". These various strands combine into a deluge of data and one of the great challenges lies in handling and distilling this. The processing capabilities of the designer's brain are limited, yet it can be exposed to vast amounts of information: airframe dimensions, shapes, topologies, loads, performance and quality metrics, cost analyses, certification criteria, etc.

In addition, two types of higher level information are required to make sense of all this. First, a trustworthiness label needs to be attached to each data item - a typical issue here is understanding the level of fidelity provided by an analysis tool. Secondly, the intricate web of relationships between the various sources of information must be known and feedback loops must be built into the design

*Research Fellow, Computational Engineering and Design Group, AIAA Member.

${ }^{\dagger}$ Professor of Computational Engineering, Chair of Computational Engineering and Design Group.

1

American Institute of Aeronautics and Astronautics 
workflow accordingly. For example, any change in the current estimate of the design take-off weight might require re-alignment with the relevant certification criteria. Variations in these can have an impact on the required size of the flight envelope, which, in turn might trigger changes in the load cases applied to the structural models being considered. If, consequently, any structural members need to be resized, leading to a change in the empty weight, the choice of powerplant might have to be reconsidered, which, in turn, is correlated with the necessary wing area... We could go on, but we hopefully have, by now, made the point that the scope for iterative heuristics is virtually endless.

The above discussion applies to fixed wing aircraft design in general, but some of the challenges of Unmanned Air Vehicle (UAV) conceptual design differ slightly from those encountered by the designers of manned aircraft. UAV airframe design usually involves considerably more topological freedom for two main reasons. First, the UAV world has not yet reached the maturity that characterizes the design of, say, commercial airliners, whose shape and topology is the result of a vast amount of commercial pressure and design effort over a century of passenger air transport. Secondly, engineering a UAV is a less constrained process than producing a new manned aircraft concept. The reasons for this range from having fewer certification criteria to not having to shape the fuselage to accommodate the crew and/or passengers in a comfortable fashion.

And therein lies the difficulty. With all the advantages of this freedom (mainly in terms of life-cycle cost) comes a design space that is far larger than that normally considered by, say, the designers of passenger airliners, and it is therefore more difficult to explore in the course of the concept design process.

The work reported in this paper is aimed at constructing a multidisciplinary analysis capability suitable for searching such a design space. In implementation terms, this translates into a requirement for analysis that is computationally cheap enough to fit a large number of evaluations into the computational budget determined by the time constraints of the conceptual design cycle, while maintaining a level of fidelity that enables meaningful design trade studies.

With commercial CAD tools becoming the weapons of choice at all levels of the design process (gradually expanding from their "birthplace" in detail design up to the initial concept selection phase), we center our design philosophy around a geometry service based on such a CAD engine - this is what we discuss next.

\section{Geometry Provision}

The modeling and parameterization of airframe geometries are sciences in their own right and the reader interested in an overview of these topics may wish to consult any of a number of good surveys (e.g., Refs. 2 and 3). The geometry service at the center of the conceptual design framework discussed here is based on a generic model constructed using the CATIA ${ }^{\circledR}$ V5 R15 CAD engine. The model is built on a wireframe comprising a centrebody and a wing, where the former can be stretched in all three directions to form either fore- and aft fuselages or various blended wing-body configurations. Similarly, the wing has a sufficient number of degrees of freedom to accommodate concepts ranging 


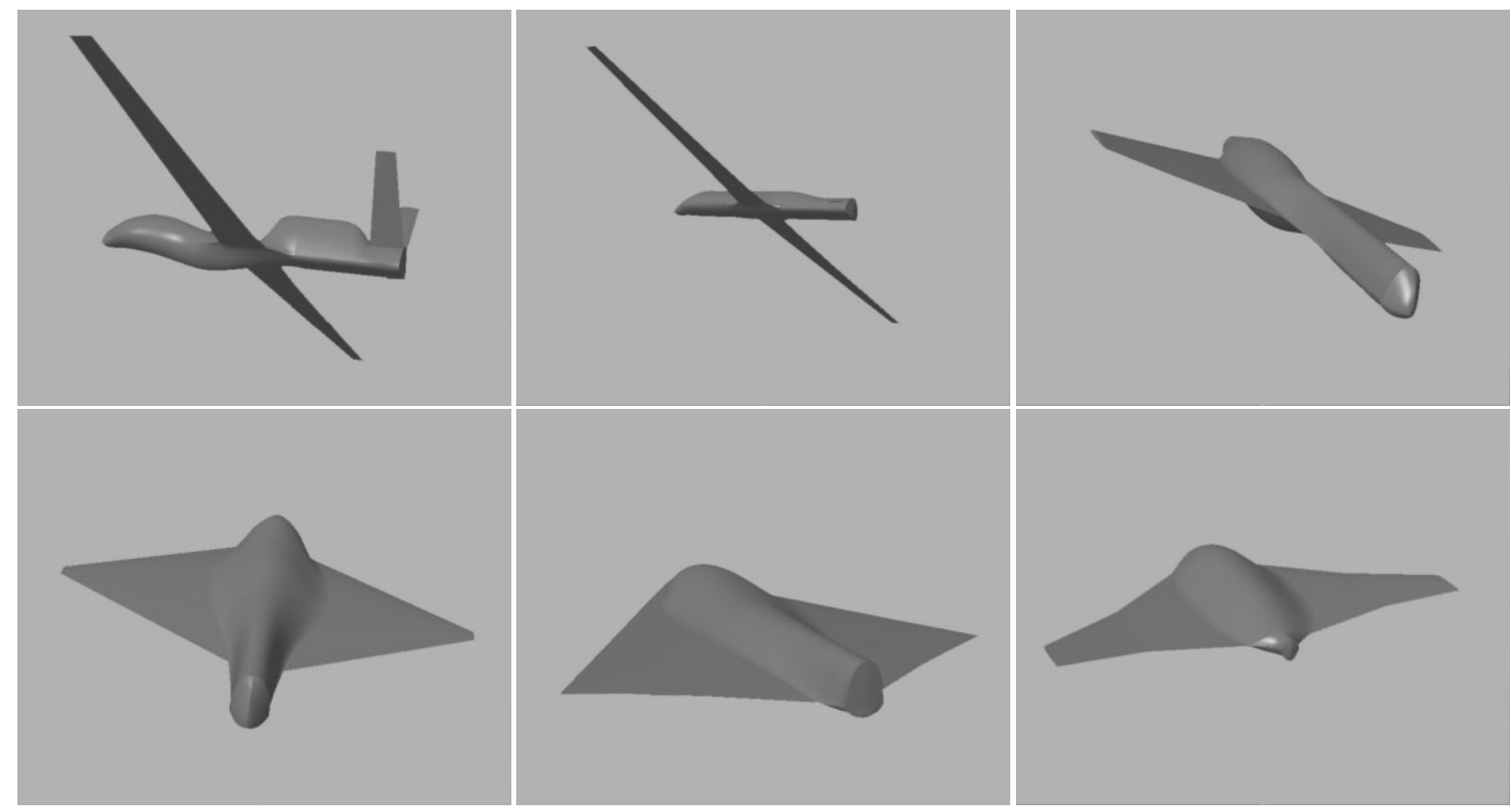

Fig. 1 A selection of geometries generated by the geometry service, including a clone of the Global Hawk (top left), morphing into an X-47 clone (bottom centre).

from long endurance type, high aspect ratio wings to short, highly loaded delta wings. Figures 1 and 2 illustrate the versatility of the external surface and internal structure CAD models respectively.

One of the noteworthy features of the structural model is that it provides a geometry that will, in most cases, prove to be over-engineered. In other words, it provides a large number of components, which, thanks to the modular nature of the model, can be switched on or off as dictated by the requirements of the problem in hand. This and other aspects of the geometry service are discussed in more detail in Ref. 4, with a more general take on geometry generation in the context of conceptual design in Ref. 5. Here we focus on the multidisciplinary design process itself, which we now turn our attention to.

\section{Design workflow}

The conceptual phase of the design process, which is our focus here, converts the requirements (the mission profile) into a concept that will serve as the baseline for the preliminary design process. This is the part of the development cycle with the widest scope - in optimization terminology, conceptual design is low-resolution global search, while the following stages have the role of increasing the resolution, localizing the search in ever smaller spaces and gradually increasing design detail.

Our design workflow (Figure 4) is thus the roadmap of a journey from mission definition to baseline concept, ready for preliminary design. We will take the reader on such a journey in Section V, where we discuss a specific design problem in some detail - for the time being, however, we briefly review the major stations along the design process, as shown in Figure 4. 

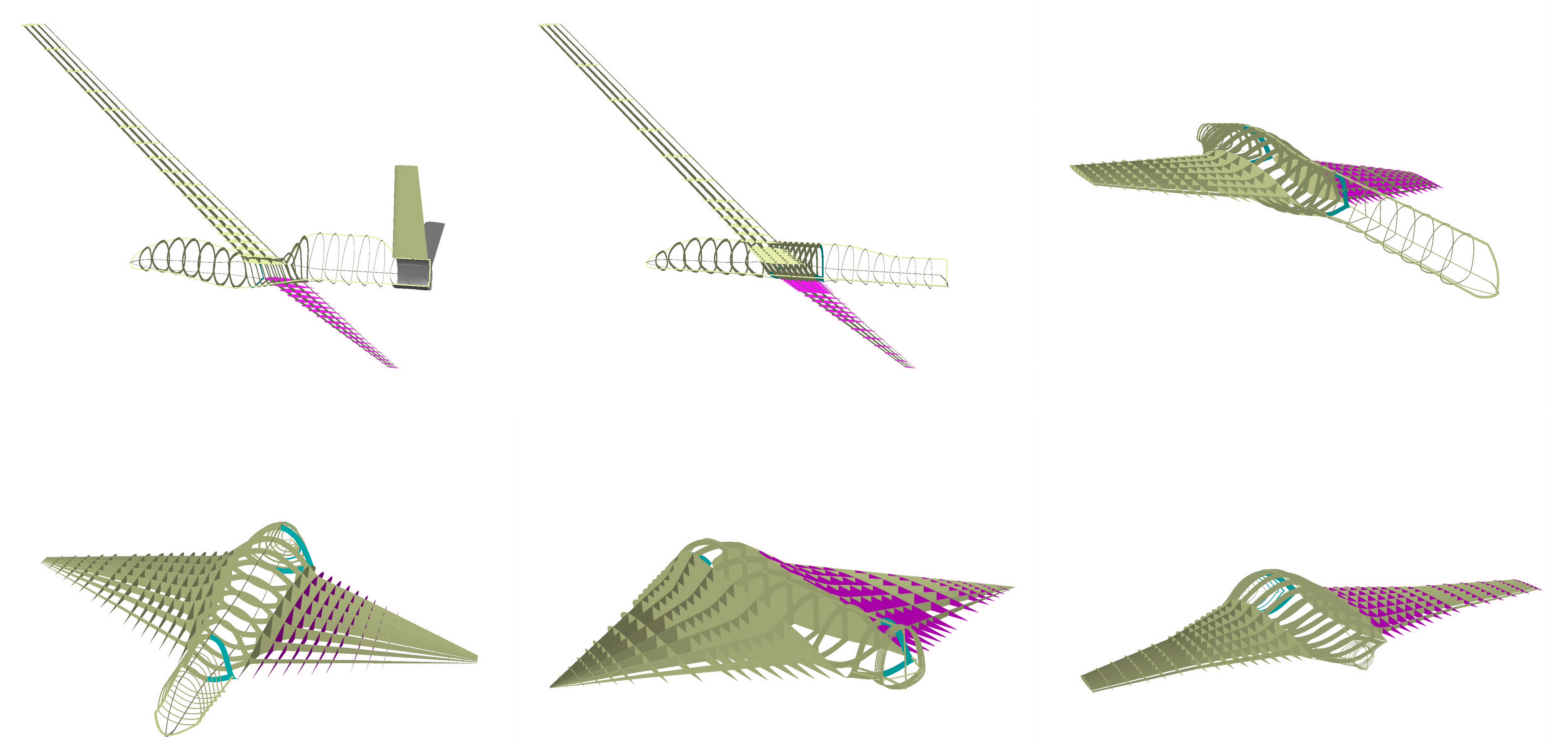

Fig. 2 A selection of internal structure models generated by the geometry service of the UAV conceptual design system, corresponding to the external surface geometry sequence shown in Figure 1.

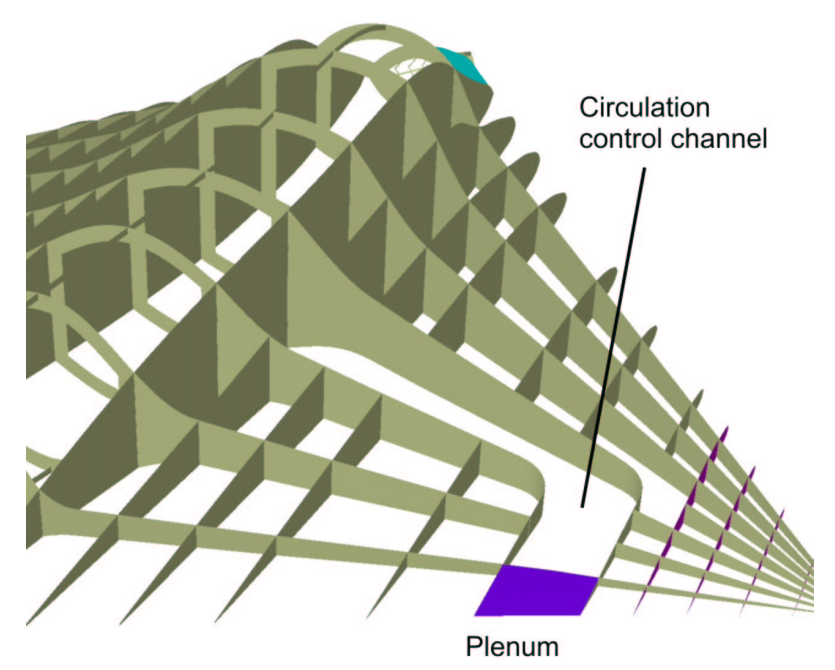

Fig. 3 An example of a modified internal structure - in this case showing the inclusion of a tunnel for a circulation control duct. 
Following Raymer ${ }^{6}$ we begin by establishing an initial airframe layout "sketch", represented by an instance of the generic CAD geometry, along with a set of design variables and their bounds, selected from all possible degrees of freedom of the generic model. This is a step that clearly requires some experience, though it is clear, for example, that an agile, fast aircraft will have short, stubby wings, while long endurance missions will demand long, high aspect ratio wings - this is the type of fairly "coarse grain" decision that has to be made at this stage.

A number of different instances are then generated with the newly restricted design variables and their wetted area / reference area ratios measured - the average figure, in conjunction with the approximate wing aspect ratio, can be used to estimate the maximum lift to drag ratio (L/D) of the aircraft.

With the $\mathrm{L} / \mathrm{D}$ value in place and an estimate of the specific fuel consumption figure typical of the class of engines we are likely to require, we can now use the Breguet range equation to compute the cruise weight fraction. This, in turn, will provide us with the first estimate of the design take-off weight of the aircraft. This is the most valuable piece of information we have at this stage and, first and foremost, we use it to establish the airworthiness criteria we will be working against in subsequent steps of the design process.

Since, at the moment, there are no specific UAV certification documents available, the task is to establish which existing (manned aircraft oriented) ruleset to use. Clearly, the strictness of the airworthiness criteria should depend on the potential of the vehicle to cause unintended harm. According to guidance issued by the UK Civil Aviation Authority (CAA), ${ }^{7}$ we assume that harm could be caused by two types of impact: as a result of an attempted emergency landing under control or as a result of complete loss of control. We compute two crash (kinetic) energy values accordingly: using the maximum take-off weight and the approach speed (emergency landing, or "unpremeditated descent") and the maximum take-off weight and the probable terminal velocity (crash after loss of control). These values are then compared against the corresponding values of existing manned aircraft and the certification bracket of similar manned aircraft is adopted (Ref. 7 offers statistical data that can be used for this purpose) $)^{*}$.

With the airworthiness criteria thus clarified, the sizing process can begin, chiefly in terms of the wing area. An example of the classic constraint analysis leading to a wing area estimate will be provided in Section V. - for now we note that the performance figures estimated as a result of this must be checked against the certification criteria established earlier.

The generic CAD model at the centre of the tool can now be scaled to the dimensions obtained here, i.e., it can be promoted from a statement of morphology (our initial planform choice) to an actual design (tentative as it may be at this stage). The internal structure can also be tailored to our requirements, by removing any unnecessary elements, thus readying our model for the first iterations

\footnotetext{
*It is possible that two different brackets will result from the two comparisons. In such cases any feature of the design affecting the ability of the vehicle to maintain a safe altitude will be designed for the criteria found from the first calculation and the document chosen from the second calculation will be used to assess the airworthiness aspects of the design that affect the ability of the pilot to maintain control of the aircraft.
} 


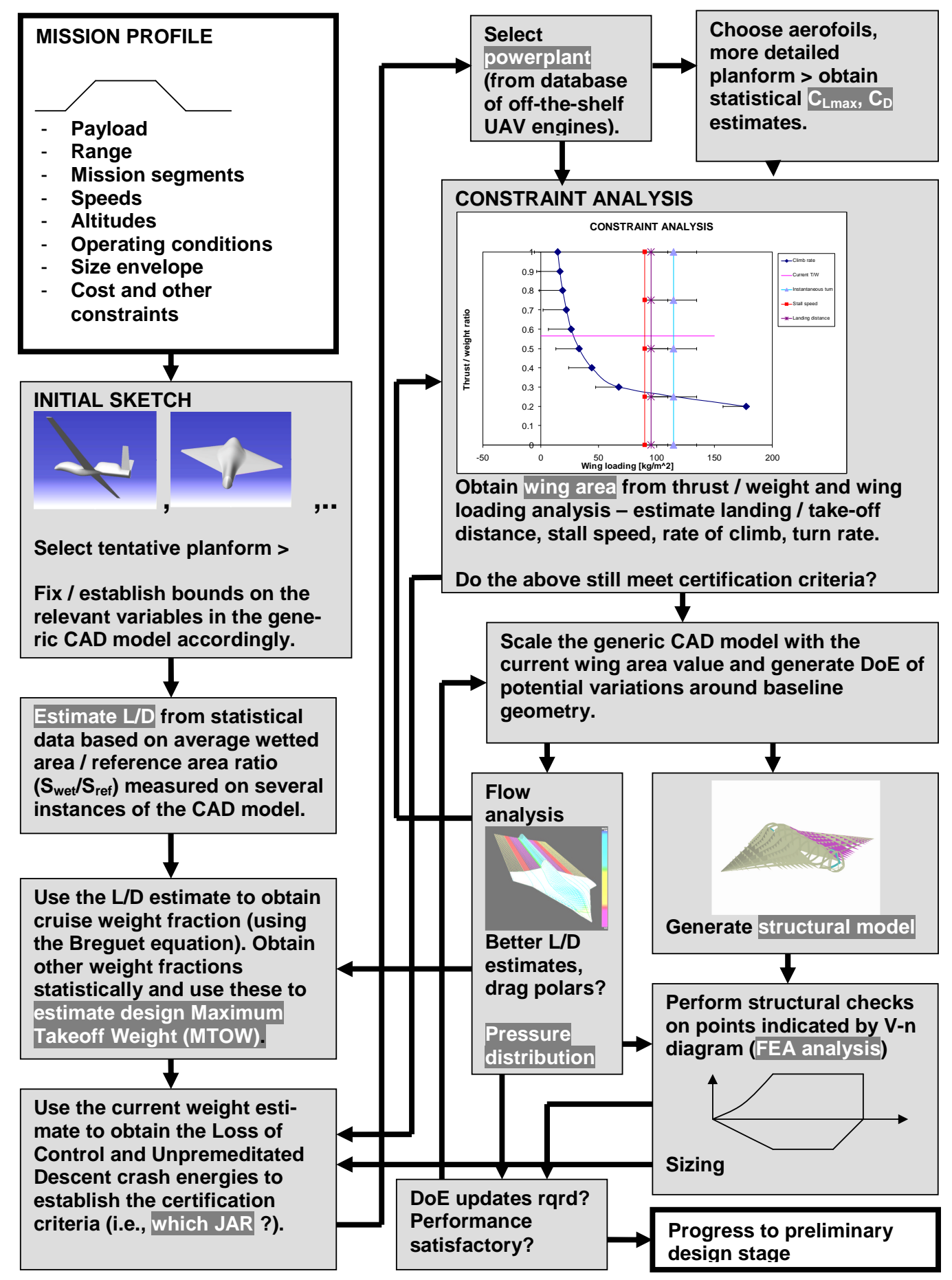

Fig. 4 Conceptual design workflow.

6 
of multidisciplinary analysis (this will be discussed in more detail in Section IV).

At this stage it is also advisable to select the design variables we will be using for any subsequent trade-off studies - this will be a subset of those describing the generic model. As we hinted earlier, in geometry definition terms, choosing a planform and general shape and topology is, in fact, equivalent to freezing a number of the initial design variables and establishing bounds on the remaining ones. With these bounds in place, a design of experiment ${ }^{8}$ can be built, which can form the basis of any subsequent multidisciplinary optimization or "what if" studies.

\section{Multidisciplinary Analysis}

As mentioned in the introduction, there is, inevitably, a computational expense constraint on the analysis capability built into a conceptual design tool. After all, very few "what if" type studies can be feasibly conducted if an answer requires days of computing time. Moreover, in most conceptual design studies the models themselves are not detailed enough to warrant the use of a very sophisticated analysis capability - the design tool discussed here is no exception to this.

Beyond the challenge of avoiding such mismatches and managing the fidelity versus computational cost trade-off, the next challenge is to combine the various strands of the multidisciplinary analysis capability. This is a difficult integration excercise for several reasons: there is a complex set of couplings between the disciplines, the computational costs of the various disciplines can be different, etc. (see Keane and Nair ${ }^{3}$ for a detailed discussion). A sizeable body of literature is available on such integrated systems, see, for example, reports on the MOB project (e.g. La Rocca et al. ${ }^{9}$ and Österheld et al. $\left.{ }^{10}\right)$, the interactive system of Liu et al., ${ }^{11}$ the CAFFE framework ${ }^{12}$ or a recent survey of various schemes by Perez. ${ }^{13}$

Here we opted for a loose coupling between the components, allowing the user to call upon the analysis tools in a manner that best suits their engineering thought processes. The workflow shown in Figure 4 is one possible structure, though the modules represented by the rectangular boxes can be wired in other ways if desired. Of course, certain elements of the workflow are fixed. For example, flow analyses, which we typically perform using the panel-based linearized potential flow solver VSAERO ${ }^{\circledR},{ }^{14}$ must be preceded by a patch- and panel generation step (we use the surface meshing capability within GridGen ${ }^{\circledR}$, driven by its built-in Glyph ${ }^{\circledR}$ scripting engine).

In its current version, the structural analysis strand of the tool is based on ABAQUS ${ }^{\circledR} .{ }^{15}$ The structural part of the CATIA geometry (discussed in Section II) is transferred to ABAQUS via a STEP description. A shell model is then built, meshed and solved in ABAQUS (driven by a Python script). The loads result from the pressure distribution computed by VSAERO, which are scaled to reflect the corner of the boundary of the flight envelope being explored (more on this through a specific example in the next section). This symbiosis of the two strands of the analysis process is illustrated by Figure 5, a schematic of the pressure coefficient distribution on the external surface of a blended wing-body aircraft and the von Mises equivalent stresses given rise by it in the internal structure.

Of course, other analysis tools can be integrated into the system, provided that the geometry 


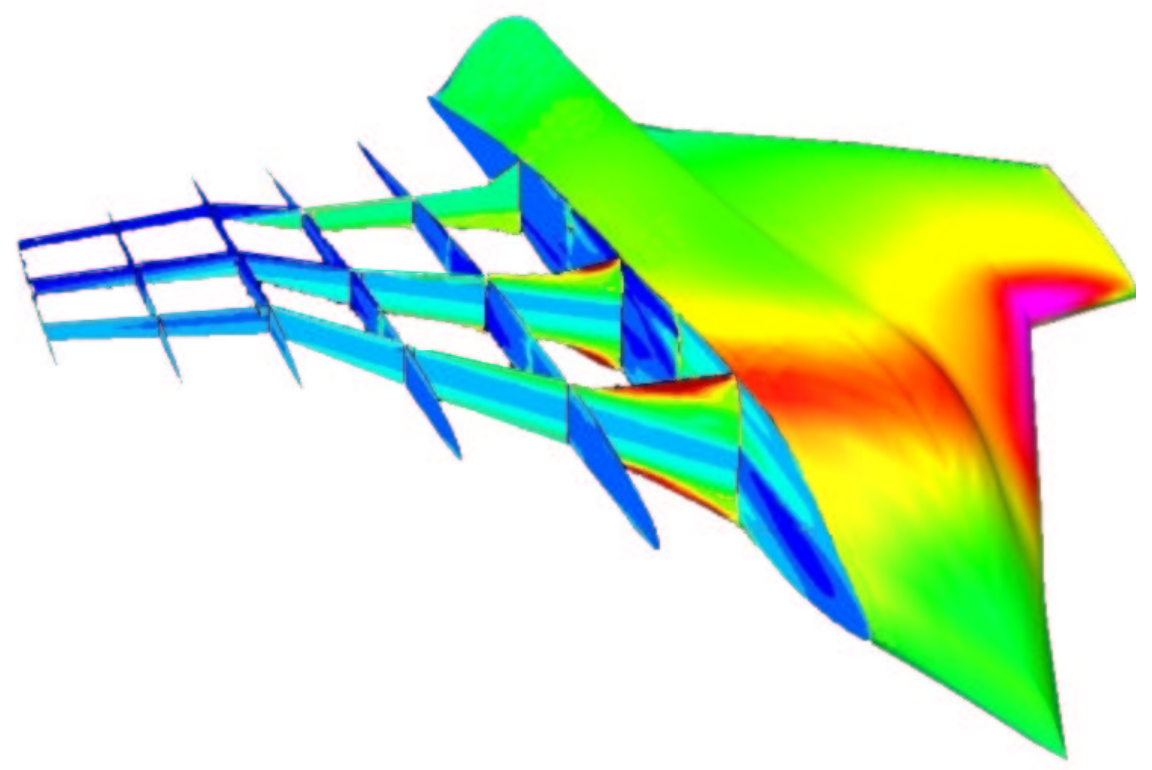

Fig. 5 Composite schematic illustrating the stress distribution in the internal structure of an aircraft model, as well as the pressure distribution on the external surface that gave rise to these stresses.

transfer challenges are overcome (for example, an earlier version of the system presented here was based on a Fluent ${ }^{\circledR}$ flow analysis capability and the CATIA ELFINI ${ }^{\circledR}$ structural solver).

\section{A Blended Wing-Body Design Study}

We now illustrate the design workflow discussed above through an example based on the following notional mission profile. A UAV is to be designed to deliver two $256 \mathrm{~kg}$ missiles to a distance of $500 \mathrm{~km}$, where they are to be launched from the aircraft's cruising altitude of 10,000ft. The launch area should be reached in no more than fifty minutes. Should the aircraft be unable to engage the target, the missiles must be returned to base, where the aircraft has to be able to land with a ground roll of no more than $700 \mathrm{~m}$ and is required to have a fuel reserve of $10 \%$ of the total mission fuel.

According to the workflow shown in Figure 4, the first step is to select an approximate airframe topology. We choose to work here with a blended wing-body instance (shown in Figure 5) of the generic geometry, having a wetted area / reference area ratio of around 2.1 and an aspect ratio of 2. Using the relevant statistical model from Ref.6, the maximum lift / drag ratio of the aircraft is expected to be around 14, which, considering the required endurance and the thrust specific fuel consumption (TSFC) value from a tentative engine choice (more on which shortly), gives a cruise fuel fraction of 0.891. Using this, as well as a database of UAV fuel weight fraction data (compiled specifically for the purposes of this design tool) we arrive at an initial MTOW estimate of $1,587 \mathrm{~kg}$.

We can now select the relevant set of certification criteria following the guidance issued by the CAA. As discussed before, this is based on the crash energy of the aircraft in two scenarios: unpremeditated 8 
descent and loss of control. The model provided by the CAA places the aircraft under the authority of 14 CFR Part 23/JAR-23 for both cases.

An empirical model based on the same UAV database is used to estimate the thrust requirements of the aircraft, which, in this case, place us in the 2,000lbf thrust class. A representative engine, which we tentatively choose at this stage is the Williams FJ44-1 turbofan ${ }^{\dagger}$.

The final piece of information we require before conducting the constraint analysis is an estimate of the aerodynamic performance of the aircraft. For the purposes of this study we choose a NACA 1802 airfoil to base our wing geometry on and, for an estimate of the lift curve slope and the maximum lift coefficient, we run a model developed by the Engineering Sciences Data Unit. ${ }^{16}$

With these estimates in place we draw up the constraint diagram represented in Figure 6 . It is generally accepted that, unless high maneuvrability is required, cost and weight should be minimized by choosing a high wing loading value (i.e, a small wing). According to the constraint analysis it is clearly the stall speed requirement that constitutes the active constraint and, at $56 \mathrm{~kg} / \mathrm{m}^{2}$ it is considerably less than the wing loading of $95 \mathrm{~kg} / \mathrm{m}^{2}$ required by the next constraint, that of the landing distance.

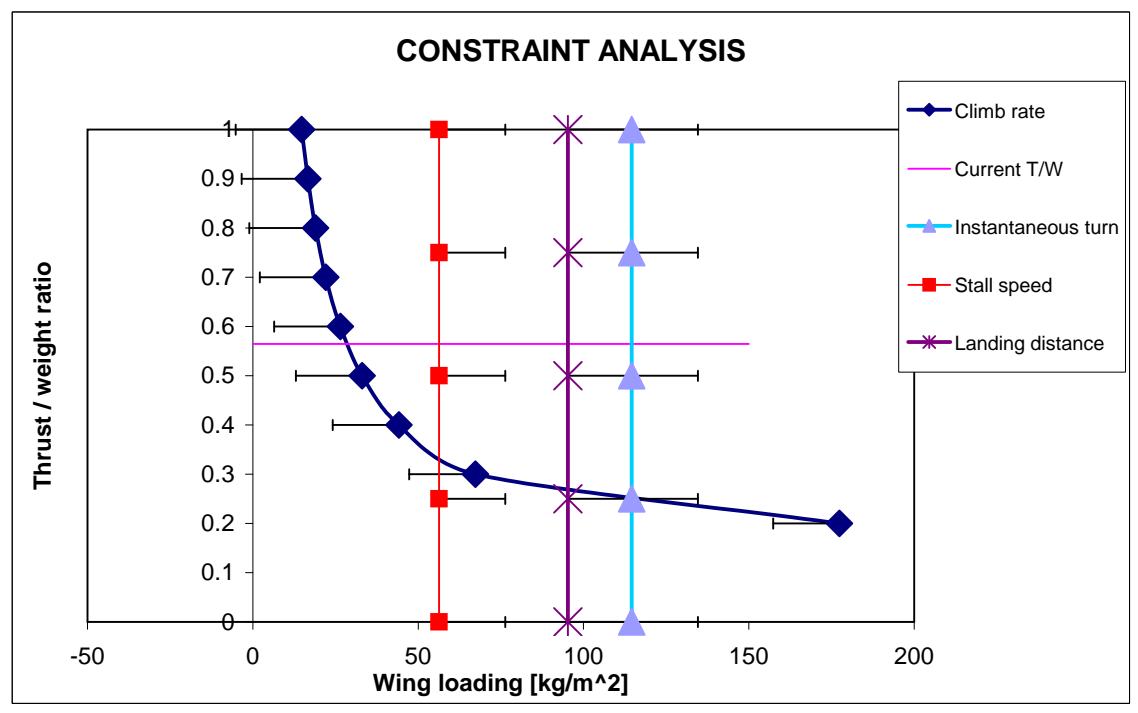

Fig. 6 Constraints after the first design iteration.

The only way around this is to place the aircraft in a different certification bracket, by revisiting our initial choice of powerplant. 14 CFR Part 23/JAR-23 limits the stall speed of single engine aircraft to no more than $61 \mathrm{kts}$ - replacing the 2,000lbf thrust class Williams turbofan with two 1,000lbf thrust class Teledyne Continental F408-CA-400 turbojets will lift this constraint ${ }^{\ddagger}$. With their external diameter of $0.335 \mathrm{~m}$ each, our tentative airframe can accommodate the two engines

\footnotetext{
${ }^{\dagger}$ Note that, since a TSFC value was required for the cruise fuel fraction calculation, that will need to be re-checked if a different engine is selected here and thus some iteration may be required at this stage.

${ }^{\ddagger}$ Provided, of course, that critical engine inoperative climb requirements are met - this will have to be checked at the preliminary design stage.
}

9

American Institute of Aeronautics and Astronautics 
in a vertical (English Electric Lightning type) arrangement. Increasing the flaps-up stall speed to $77 \mathrm{kts}$, the maximum feasible wing loading is now $90 \mathrm{~kg} / \mathrm{m}^{2}$, as shown in Figure 7. This gives us a wing reference area of $17.64 \mathrm{~m}^{2}$ (as opposed to the value of $28.34 \mathrm{~m}^{2}$ that the initial, single engine configuration would have given us).

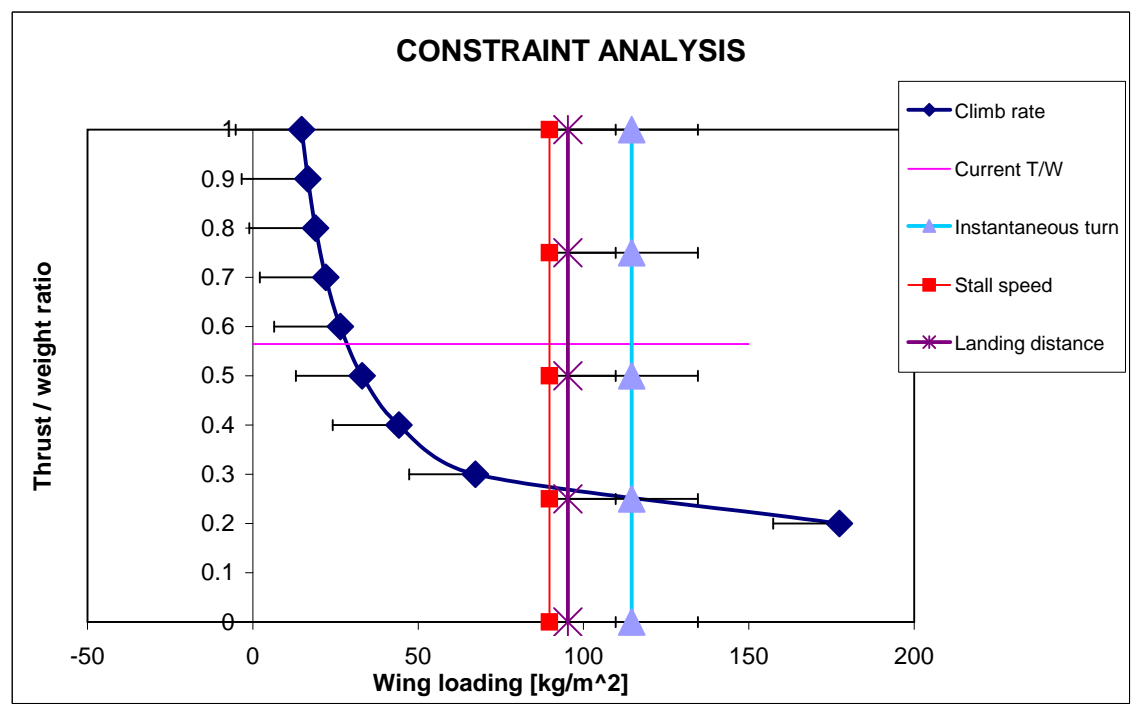

Fig. 7 Constraints after the second design iteration.

The CAD model can now be scaled to this wing area value, giving a span of $5.84 \mathrm{~m}$.

We are now ready to conduct multidisciplinary trade-off studies on our candidate design. We illustrate such a process here by experimenting with three different values of negative twist and two values of outboard sweep angle (i.e., the sweep of the outboard section of the wing) in a full factorial experiment - these variables are illustrated in Figure 8. Large washout angles can clearly be beneficial from the perspective of handling and aerodynamics, but what will their effect be on the demands placed on the airframe structure?

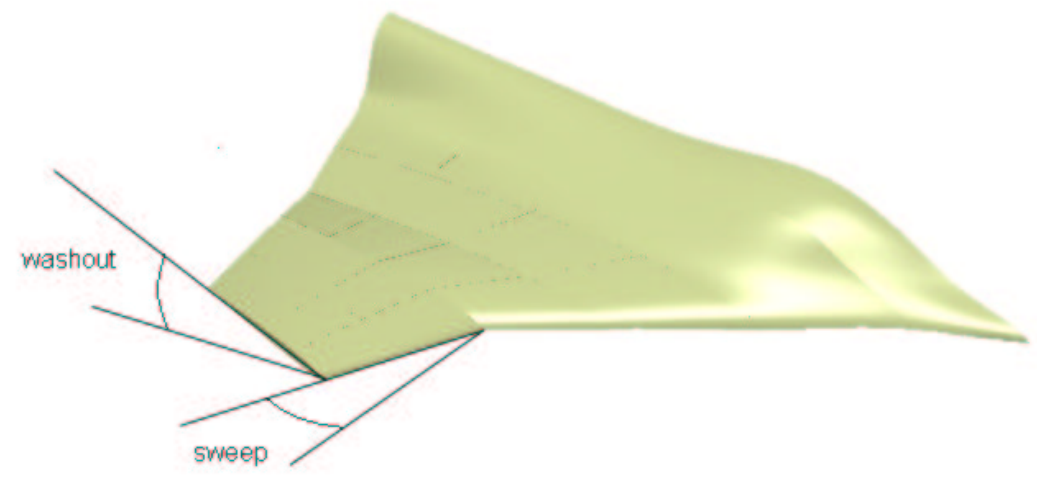

Fig. 8 Design variables used in the MDO case study.

Figure 9 depicts the flight envelope resulting from the performance estimates of our aircraft, 10 
following the airworthiness requirements established earlier. This provides the basis of our MDO study. Specifically, we will be checking each airframe at the design points marked on the V-n diagram: at the top of the accelerated stall curve and at the dive recovery condition, both at a load factor of 3.8. We obtain the required pressure distributions by running VSAERO simulations at both conditions, using the equivalent (compressibility-corrrected) airspeeds indicated on the V-n diagram. We run an additional set of VSAERO simulations for each design at cruise conditions with a range of angles of attack to obtain their maximum lift to drag ratio.

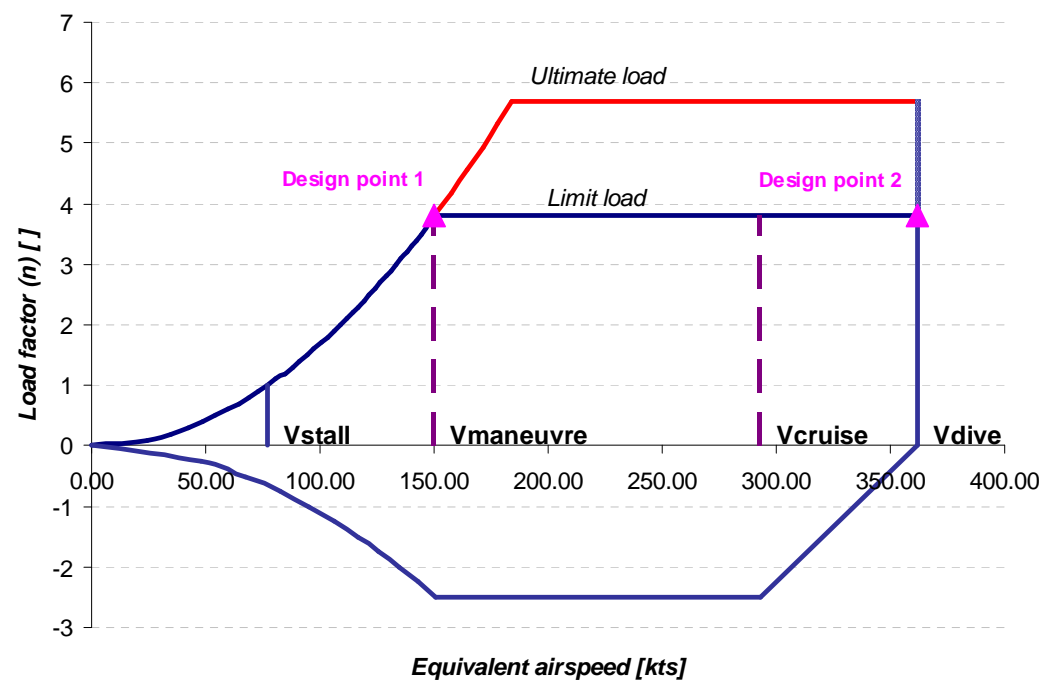

Fig. 9 UAV flight envelope. The structure is assessed at the high angle of attack / maneuvering speed condition (Design point 1) and at the low angle of attack / dive speed condition (Design point 2).

Figure 10 depicts the results of six such MDO iterations, by showing the maximum wingtip deflections versus the maximum $\mathrm{L} / \mathrm{D}$ values. We display the larger wingtip deflection resulting from the two cases (design points 1 and 2, as per Figure 9) - the decisive case is the accelerated stall in four cases and the dive recovery in the remaining two. We note here that the deflections are very small, indicating that the airframe is over-engineered, in terms of part sizes and / or numbers. However, at this stage we are merely interested in the relative stiffnesses offered by the different candidate designs - the preliminary design phase will have to improve on the initial guesses on actual rib / spar / frame / cover sizes and numbers.

Clearly, the design with a washout of $5^{\circ}$ and an outboard section sweep of $20^{\circ}$ is the best, both in terms of airframe stiffness and in terms of aerodynamic efficiency.

This small study is merely an illustration of our proposed conceptual design workflow - similar, larger scale studies could be conducted until the designer is satisfied that the best solution has been found. "The best" is, in this case, qualified as: given the resolution of the search, the fidelity of the models and the time allocated to the conceptual search within the design cycle.

11

American Institute of Aeronautics and Astronautics 


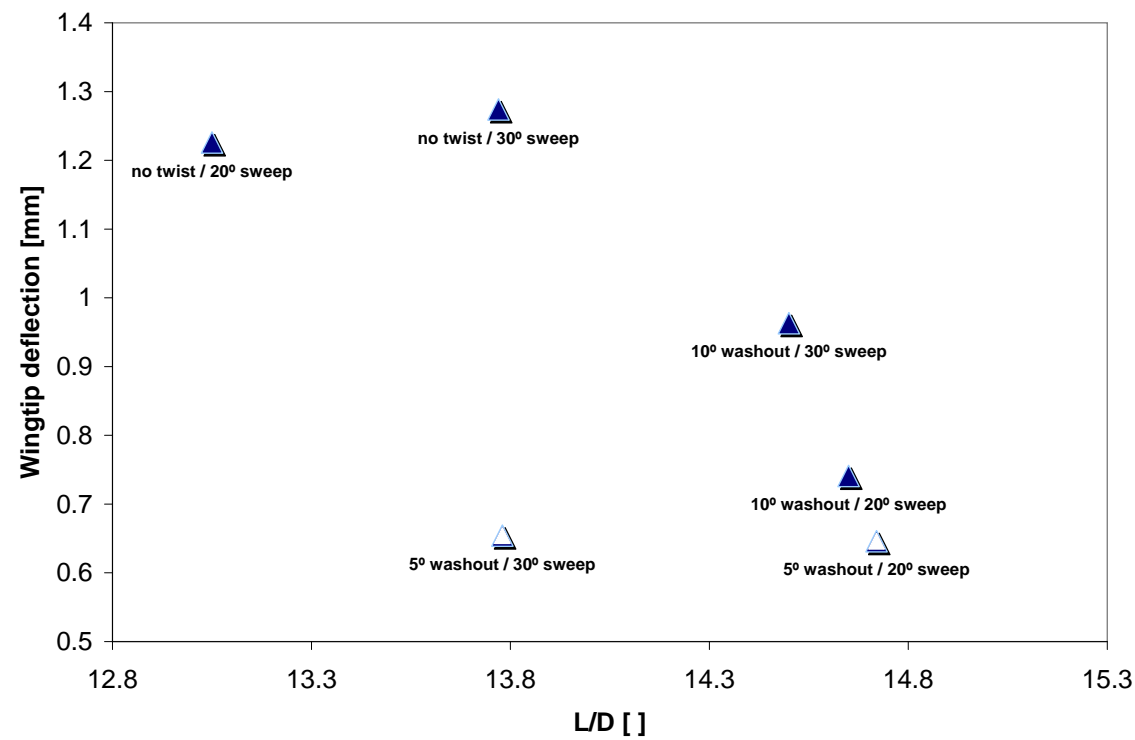

Fig. 10 The design with a washout of $5^{\circ}$ and an outboard section sweep of $20^{\circ}$ is the best on both objectives. This is one of the two designs indicated by white triangles, meaning that their active constraint is the dive condition.

\section{Conclusions}

A UAV conceptual design system has been described, illustrated and demonstrated through a specific design case study. We have shown that commercial, off-the-shelf CAD tools can be integrated into the design process as early as the conceptual level, where, as parametric geometry engines, they can take the important role of providing the models required by the various strands of multidisciplinary analysis. This permits the use of CFD and FEA solves at the earliest stages of design.

\section{Acknowledgements}

This work has been jointly supported by BAE Systems and the Engineering and Physical Sciences Research Council (United Kingdom) as part of the Integrated Programme of Research in Aeronautical Engineering.

\section{References}

${ }^{1}$ Stinton, D., The Design of the Aeroplane, Collins Professional and Technical Books, 1985.

${ }^{2}$ Samareh, J. A., "Survey of Shape Parameterization Techniques for High-Fidelity Multidisciplinary Shape Optimization," AIAA Journal, Vol. 39, No. 5, 2001, pp. 877-883.

${ }^{3}$ Keane, A. J. and Nair, P. B., Computational Approaches to Aerospace Design: the pursuit of excellence, John Wiley \& Sons., 2005.

${ }^{4}$ Sóbester, A., Keane, A. J., Scanlan, J., and Bressloff, N. W., "Conceptual Design of UAV Airframes Using a Generic Geometry Service," AIAA Infotech@Aerospace, Arlington, VA, 2005.

${ }^{5}$ Sobester, A. and Keane, A. J., "Supervised Learning Approach to Computer-Aided Design Geometry Repair," AIAA Journal, Vol. 44, No. 2, 2006, pp. 282-289. 
${ }^{6}$ Raymer, D. P., Aircraft Design: a Conceptual Approach, AIAA Education Series, American Institute of Aeronautics and Astronautics, Washington DC, 3rd ed., 1999.

${ }^{7}$ Haddon, D. R. and Whittaker, C. J., "Aircraft Airworthiness Certification Standards for Civil UAVs," Proceedings of UAV Systems: Shaping the 21st century, Royal Aeronautical Society, London, 2005, pp. 1-15.

${ }^{8}$ Sacks, J., Welch, T. J., and Wynn, H. P., "Design and Analysis of Computer Experiments (with discussion)," Statistical Science, Vol. 4, 1989, pp. 409-435.

${ }^{9}$ La Rocca, G., Krakers, L., and van Tooren, M. J. L., "Development of an ICAD Generative Model for Blended Wing Body Aircraft Design," 9th AIAA/ISSMO Symposium on Multidisciplinary Analysis and Optimisation, Atlanta, 2002 .

${ }^{10}$ Osterheld, C., Heinze, W., and Horst, P., "Preliminary Design of a Blended Wing Body Configuration using the Design Tool PrADO," CEAS Conference on Multidisciplinary Aircraft Design and Optimisation, Koln, 2001.

${ }^{11}$ Liu, H., Wang, G. L., Lu, X. L., and Wu, Z., "Preliminary Investigation of Integrated Multidisciplinary Optimization in Aircraft Conceptual Design," 10th AIAA/ISSMO Multidisciplinary Analysis and Optimization Conference, Albany, 2004.

${ }^{12}$ Antoine, N., Kroo, I., Willcox, K., and Barter, G., "A Framework for Aircraft Conceptual Design and Environmental Performance Studies," 10th AIAA/ISSMO Multidisciplinary Analysis and Optimization Conference, Albany, 2004.

${ }^{13}$ Perez, R. E., Liu, H. H. T., and Behdinan, K., "Evaluation of Multidisciplinary Optimization Approaches for Aircraft Conceptual Design," 10th AIAA/ISSMO Multidisciplinary Analysis and Optimization Conference, Albany, 2004.

14 "www.am-inc.com/VSAERO.shtml," Accessed 1 May 2006.

15 "www.abaqus.com/products/products_cae.html," Accessed 1 May 2006.

16 "Program for Calculation of Maximum Lift Coefficient of Plain Aerofoils and Wings at Subsonic Speeds," Data Item 93015, Engineering Sciences Data Unit, 1993. 